\title{
Neutrophil Gelatinase Associated Lipocalin: Is not an Early Marker Inductor for Diabetic Nephropathy in Qatari Population
}

\author{
Elham Sharif* and Mariam Alwakeel \\ Department of Biomedical Sciences, College of Health Science, Qatar University, Qatar
}

*Corresponding author: Elham Sharif, Department of Biomedical Sciences, College of Health Sciences, Qatar University, Po box 2713, Doha Qatar

\begin{abstract}
ARTICLE INFO
Received: 幽 September 29, 2019

Published: October 16, 2019

Citation: Elham Sharif, Mariam Alwakeel. Neutrophil Gelatinase Associated Lipocalin: Is not an Early Marker Inductor for Diabetic Nephropathy in Qatari Population. Biomed J Sci \& Tech Res 22(1)-2019. BJSTR. MS.ID.003692.
\end{abstract}

Keywords: Diabetes Mellitus; Diabetic Nephropathy; Neutrophil Gelatinase Associated Lipocalin; Lipocalin-2

Abbreviations: DM: Diabetes Mellitus; T2DM: Type 2 Diabetes Mellitus; DN:Diabetic Nephropathy; NGAL: Neutrophil Gelatinase Associated Lipocalin; uNGAL: Urinary Neutrophil Gelatinase Associated Lipocalin; SCr: Serum Creatinine; BUN: Blood Urea Nitrogen; eGFR: Estimated Glomerular Filtration Rate; ANOVA: Analysis of variance

\section{ABSTRACT}

Background: The WHO Global Report on Diabetes (2016) showed that the number of diabetic patients quadrupled between 1980 and 2016, while causing the death of 1.5 million people. While the global prevalence of diabetes is $9 \%$, the prevalence of diabetes in Qatar is between $17-20 \%, 45 \%$ of which developed diabetic nephropathy. Diabetic Nephropathy is the largest cause of End Stage Renal Disease, and it develops in 20\% of diabetic patients. Currently, DN is diagnosed by the detection of microalbumin in urine samples. However, nephropathy can be present even in the absence of albuminuria, and the levels of microalbumin in urine does not correlate with the degree of nephropathic damage. Early detection can prevent total renal failure. Studies have shown that neutrophil gelatinase-associated lipocalin (NGAL) was highly expressed even before the appearance of pathological microalbuminuria in both type 1 and type 2 diabetic patients. The levels of NGAL in urine also correlates with the degree of nephropathic damage. However, currently no information exists about the presence of NGAL in diabetic patients of the Qatari population.

Objective: This study aims to determine if there is a relationship between the concentrations of NGAL in urine and kidney function.

Methodology: Urine samples of 123 patients were acquired from the Qatar Biobank. Of these, 38 were non-diabetic controls, while 85 were diabetic patients. Type 1 diabetics, pregnant females, smokers, and kidney, liver and cardiovascular disease patients were excluded from the control and case population. Using Enzyme linked immunosorbent assay (ELISA), all samples were tested for the presence of NGAL, and a select few were also tested for microalbumin through an external laboratory. The results obtained were analyzed using Statistical Package for Social Sciences (SPSS) version 24 and Microsoft Excel 2016.

Results: No significant difference was found in mean values of UNGAL concentrations in healthy patients, diabetic patients with $\mathrm{HbA1}>>6 \%$ and diabetic patients with HbA1c $<6 \%(p>0.05)$. However, weak correlation was demonstrated between uNGAL concentrations with serum albumin, $\mathrm{HbA} 1 \mathrm{c}$, serum glucose concentration and albuminuria in diabetic patients with $\mathrm{HbA1}>6 \%(\mathrm{p}<0.05)$.

Conclusion: According to the current study uNGAL concentrations does not correlate with any of the kidney function tests, such as glomerular filtration rate, serum creatinine and blood urea nitrogen. So, it cannot be used as a marker to detect diabetic nephropathy in the early stages

\section{Introduction}

According to the 2017 report of the International Diabetes Federation, about $16.5 \%$ of the adult population of Qatar is expected to be diabetic, while 513 deaths are estimated to be due to diabetes [1]. A 2012 survey carried out by the Supreme Council of Health 
showed that about $17-20 \%$ of the Qatari population was diabetic, while $45 \%$ of them developed Diabetic Nephropathy (DN) [2]. DN is a microvascular complication of diabetes, and it is clinically characterized by persistent albuminuria, decline in glomerular filtration and function, and high risk of cardiovascular mortality and morbidity. DN, which is one of the microvascular complications of diabetes, is the largest cause of End Stage Renal Disease (ESRD) [3]. A kidney biopsy is the most accurate way of diagnosing DN, but since it is an invasive procedure, currently, DN is mainly diagnosed by testing the concentration of urine microalbumin. However, often, there is normoalbuminuria even though glomerular damage is observed under the microscope. In some laboratories, the serum creatinine and blood urea nitrogen [4], and the estimated glomerular filtration rate is also used as a diagnostic tool. However, these parameters are affected by various other physiological and pathological factors, such as pregnancy, exercise, dehydration, cardiovascular disease and inflammation, to name a few.

Moreover, the above-mentioned criteria often manifest only after the disease has progressed. Since early detection is the key to manage nephropathy patients, it is important to determine a suitable biomarker that is detectable in the earliest stages of DN. Neutrophil Gelatinase associated lipocalin (NGAL) is one such biomarker [5]. It is secreted when there is damage to tubular cells, which precedes glomerular damage, and is therefore seen in urine only in case of injury, and much earlier than the glomerular markers currently used. It also correlates with the degree of nephropathic damage [6]. Therefore; it is a promising biomarker to detect $\mathrm{DN}$ in the early stages to facilitate efficient patient management [7]. Based on previous publications, it is hypothesized that the Neutrophil gelatinase associated lipocalin (NGAL) is an early diagnostic biomarker of diabetic nephropathy. The aim of this study was to investigate the relationship between the concentration of neutrophil gelatinase associated lipocalin with kidney function.

\section{Epidemiology of Diabetes}

Diabetes mellitus (DM) is a leading cause of mortality and morbidity [8]. It is a common disease in Qatar. According to Hamad Medical Corporation [HMC] (2017), the prevalence of diabetes is $17 \%$ and $11-23 \%$ are at risk of having diabetes [2]. Moreover, Qatar is considered to be one of the countries that have an increased rate of glucose intolerance in $17 \%$ of its population [9]. Furthermore, in 2012, it was reported that the prevalence of Qatari Type 2 Diabetes mellitus (T2DM) patients was $16.7 \%$, and by 2050 , it is predicted to reach $24 \%$, where most of T2DM are aged from 18 to 64 years old. Additionally, the prevalence of physical inactivity, obesity and active smoking were $45.9 \%, 41.4 \%$ and $16.4 \%$ respectively [9]. Obesity is considered to be a key factor that affects two-thirds of the incidence of T2DM Qatari patients. Therefore, evaluating the future of T2DM in Qatar is crucial to be notified to control the prevalence of the disease by addressing new preventive methods, early detection of the disease and therapeutic interventions [2]. Moreover, it is a worldwide metabolic disease in which its prevalence is rising to more than one million new cases annually in the USA [8]. T2DM incidence differs significantly from one geographical area to another according to the variation of environmental, lifestyle and genetic risk factors [10]. The prevalence of T2DM in adult patients is expected to rise in the next decades and to increase greatly in developing countries [11].

\section{Novel Biomarkers - Neutrophil Gelatinase-Associated Lipocalin (NGAL)}

It has been observed that the renal tubules, and especially the proximal tubules, play an important role in the development of $\mathrm{DN}$, and this lead to finding that several tubular factors can be found in the urine (tubular proteinuria) even before the onset of glomerular damage or the appearance of microalbuminuria [12]. Neutrophil gelatinase-associated lipocalin (NGAL), a member of lipocalin family has generated a great interest to be a novel marker in the detection of diabetic nephropathy in its early stages [13]. It was initially identified by Allen and Venge in 1989 from human neutrophils [14]. It is not produced by just one cell type like many other endogenous biomarkers as many different pathologies can provoke the production and release of NGAL, such as inflammation, cardiovascular diseases and others $[15,16]$. It is also called lipocalin-2 [17], and it has a barrel shaped tertiary structure that binds to small lipophilic molecules [18]. Siderophores, small iron binding molecules, are the major ligands for NGAL. It is identified as a $25-\mathrm{kDa}$ protein that exists in monomeric, dimeric and heteromeric forms. It covalently associates with human matrix metalloproteinase 9 (MMP-9) from human neutrophils that is stored mainly in the specific granules of neutrophils [19]. It protects it from degradation.

\section{Functions of NGAL}

NGAL is expressed in very minimal levels in several human tissues, such as kidney, trachea, lungs, stomach, and colon. It is found to possess diverse functions such as transportation, activation of MMP-9, induction of apoptosis, tumourgenesis, and regulation of immune responses. NGAL also plays a renoprotective role through enhancing tubule cell proliferation in kidney injury, especially in ischemia-reperfusion injury. In fact, it is one of the most robustly expressed proteins in the ischemic or nephrotoxic injury of kidney $[20,21]$. In physiological process, recent researches have proved that NGAL can trigger nephrogenesis by stimulating the conversion of mesenchymal cells into kidney epithelia [22]. This is because it can work as an iron-transporting protein to deliver iron, which is crucial for cell growth and development, by forming a complex with iron-binding siderophores [23]. In pathological process, accumulating evidences have suggested that NGAL relates tightly with series of renal dysfunctions.

\section{NGAL, Diabetes and Diabetic Nephropathy}

Studies have successfully investigated that the messenger RNA (mRNA) expression of NGAL is significantly higher in 
diabetic/obese human beings, and associated closely with insulin resistance and hyperglycemia, which implies that NGAL may play an important role in type-2 diabetes [12]. It is speculated that NGAL in DN is produced principally by the injured tubule cells to prevent kidney from early injury for various reasons by upregulating its mRNA within a few hours after the harmful stimuli of kidney tubules. It belongs to stress induced renal biomarkers involved in the pathophysiology of diabetic nephropathy. Firstly, since injury of renal tubule is unavoidable in the process of $\mathrm{DN}$, repair mechanisms must be started by the body. As an iron-transporting protein, NGAL may be expressed by the damaged tubule to induce regeneration since iron is necessary for re-epithelialization. Besides, the complex of NGAL/siderophore/ $\mathrm{Fe}^{2+}$ can up-regulate heme-oxygenase 1 (HO1), which could limit oxidant-mediated apoptosis of renal tubule cells through limiting iron-driven oxidant stress [23]. Second, it is well known that the pathological changes of DN involve accumulation of extracellular matrix, which is degraded mainly by matrix metalloproteinases (MMPs).

The activities of MMPs depend on metal ions and are limited by tissue inhibitors of metalloproteinase-1 (TIMP-1), and NGAL is a universal activator of the MMPs family [21]. Studies have found that the metabolic disorder in the process of DN usually destroys the balance of MMPS/TIMP: the degradation ability of MMP-9 declines and the expression of TIMP-1 is up-regulated, and therefore, extracellular matrix accumulates [12]. NGAL is capable of protecting MMP-9 from degradation [1] Moreover, NGAL can activate the MMP9 precursor directly, and counteract the inhibiting effect of TIMP-1 [12]. Thus, it is presumed based on a body of evidence that NGAL is activated to delay the progression of renal fibrosis in DN through preservation of the enzymatic activity of MMP-9 [24]. Thirdly, an expanding body of data now strongly suggests that inflammation contributes to diabetes mellitus and DN $[14,25]$. The disorder of glycometabolism stimulates the expression of inflammatory factors, and these could not only ruin the kidney tissue directly, but also provoke the secretion of type IV collagen, fibrin and others, thereby accelerating kidney sclerosis. It is suggested that NGAL acts as an immuno-modulator by binding to lipophilic inflammatory mediators like neutrophil tripeptide chemoattractant and clearing them [12]. It has also been proposed that NGAL plays a role in cell apoptosis via autocrine and paracrine pathways [26]. Thus, it contributes to the protection of diabetic kidney through restraining inflammation reactions and activating apoptosis of affected cells in the tubules and interstitium.

\section{Advantages of Using NGAL to Diagnose Diabetic Ne- phropathy}

It has been known that plasma NGAL is filtered by the glomerulus and largely reabsorbed by the proximal tubules by an efficient megalin-dependent endocytosis mechanism. Thus, the excretion of NGAL in the urine happens only when the reabsorption by proximal renal tubule is blocked due to an injury that stimulates the synthesis of NGAL. The size of NGAL must be taken into consideration as it is smaller than albumin, which explains its rapid filtration by the glomeruli and its appearance in urine of normoalbuminuric diabetic patients [26,27]. It is detected within 2-4 hours of renal injury, even before the appearance of albumin in urine [28]. It correlates with severity of renal impairment via expressing different concentrations according to the degree of chronic failure condition. It has been found that urine NGAL corresponds positively with major parameters used currently in evaluating DN and renal impairment (autosomal polycystic kidney and glomerulonephritis) such as cystatin C, blood urea nitrogen and serum creatinine [29]. In fact, it was observed by Liu et al. [30] that when diabetes was induced in rats, following the development of lesions in the kidney tubules, urine NGAL levels increased significantly earlier when compared to other biomarkers [30]. Serum NGAL increases in the very early stage of diabetic nephropathy and drops down as the disease develops. In other words, serum NGAL relates inversely with the amount of albuminuria.

It has been investigated that serum NGAL changes in this way in a variety of renal dysfunctions such as ischemia-reperfusion injury, drug- induced acute interstitial nephritis, kidney transplantation and so on. However, urine NGAL is directly related to the amount of albumin in urine. Therefore, as the disease progresses, and the functions of kidney become poorer, excretion of NGAL in urine increases day after day, the absorptive function of nephric tubule decreases, and urine NGAL reaches the highest levels in macroalbuminuric patients [31]. The amount of NGAL secreted into the urine is also correlated with the degree of kidney damage [6]. This is mainly because NGAL acts as a repair protein, and is secreted into the serum, as explained earlier. As the injury worsens, there will be leakage into the urine, which increases with disease progression. Therefore, NGAL is a promising biomarker to detect DN in the earliest stages, as it is secreted within a few hours of nephropathic damage, even before glomerular involvement. It is also demonstrative of the degree of nephropathic damage and is easily detected by non-invasive procedures.

\section{Methods and Materials}

\section{Samples and Study Population}

This research study is a cross-sectional study. Urine samples were obtained from Qatar Biobank (QBB), under the project number: QBB_IRB_Ex-2017- RES-ACC-0091-0038. The samples collected were random midstream clean-catch specimen. The case population comprised of Qatari Type 2 diabetic patients of both genders. The study was excluded Type 1 diabetics, pregnant females, smokers, and kidney, liver and cardiovascular disease patients. The control population comprised of Qatari healthy, nondiabetic individuals who weren't pregnant, smokers or patients of kidney, liver or cardiovascular diseases. Sample inclusion and exclusion are summarized in Figure 1. QBB also provided some additional clinical data of the study subjects that were used to conduct the statistical analysis. 


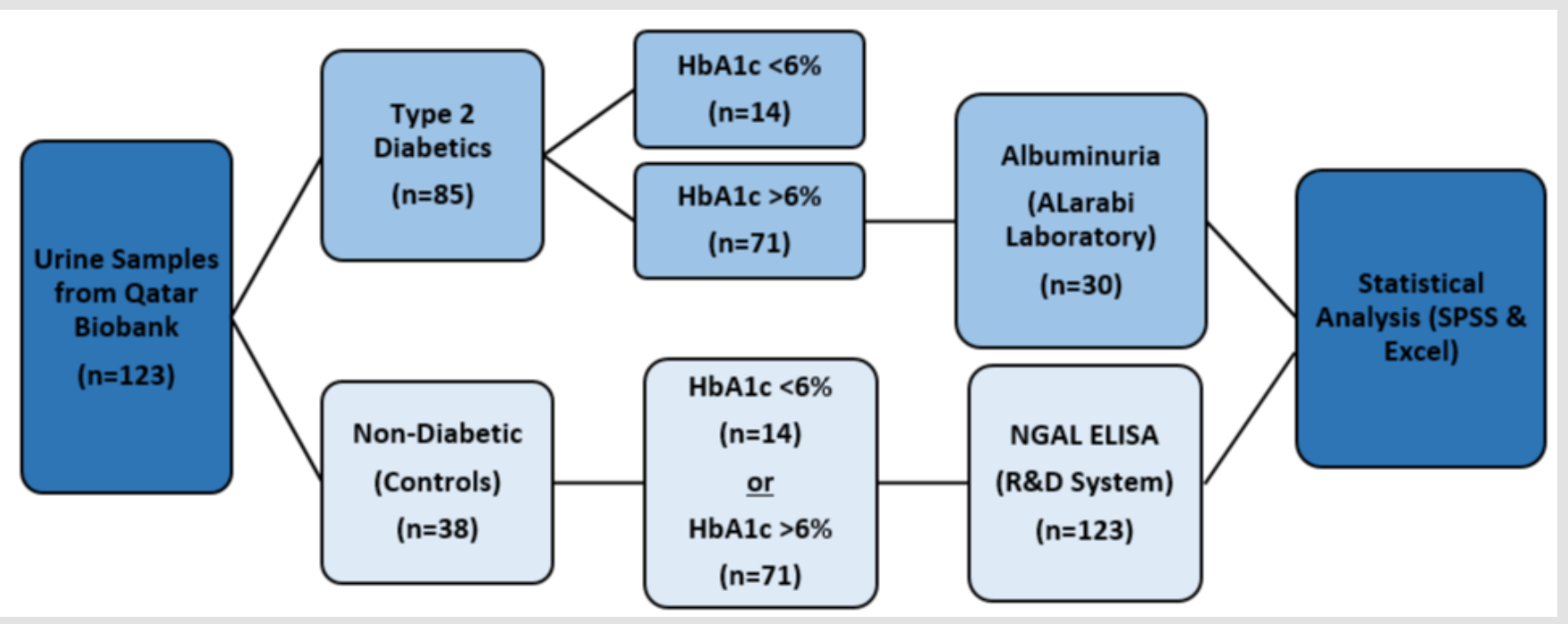

Figure 1: Flowchart summarizing methodology.

\section{ELISA for Measuring NGAL}

Enzyme-linked immunosorbent assay (ELISA) was performed using anti-human lipocalin 2 antibody (R\&D Systems, Minnesota, USA) to detect NGAL in the urine samples of both diabetic and healthy patients [32].

\section{Sample Preparation}

Centrifuged urine samples were allowed to warm to room temperature, and then used in the assay.

\section{Assay Procedure}

$100 \mathrm{uL}$ of assay diluent (provided with the kit) was added to each well. $50 \mathrm{uL}$ of standard, control or sample was added to each well, covered with adhesive strip, and incubated for 2 hours at $2{ }^{\circ} \mathrm{C}$. After incubation, an automatic washer and the prepared wash buffer was used to wash the wells four times, after which the plates were inverted and blotted against paper towels. $200 \mathrm{uL}$ of cold Human Lipocalin-2 conjugate was added to each well, and incubated for 2 hours at $2{ }^{\circ} \mathrm{C}$. After incubation, the washing process was repeated four times. Then, $200 \mathrm{uL}$ of the substrate solution was added, and incubated for 30 minutes at room temperature while protecting from sunlight. 50uL of stop solution was then added to each well. Then, within 30 minutes, the optical density was measured at $450 \mathrm{~nm}$.

\section{Analytical Technique}

Calculation of UNGAL Concentration: First, the corrected optical densities of the standards were calculated by subtracting the OD of the zero standard from the standard solutions. These values were then used to generate a 4-PL standard curve (Figure 2). Then, using the standard curve, the concentrations of NGAL in all samples and controls were calculated.

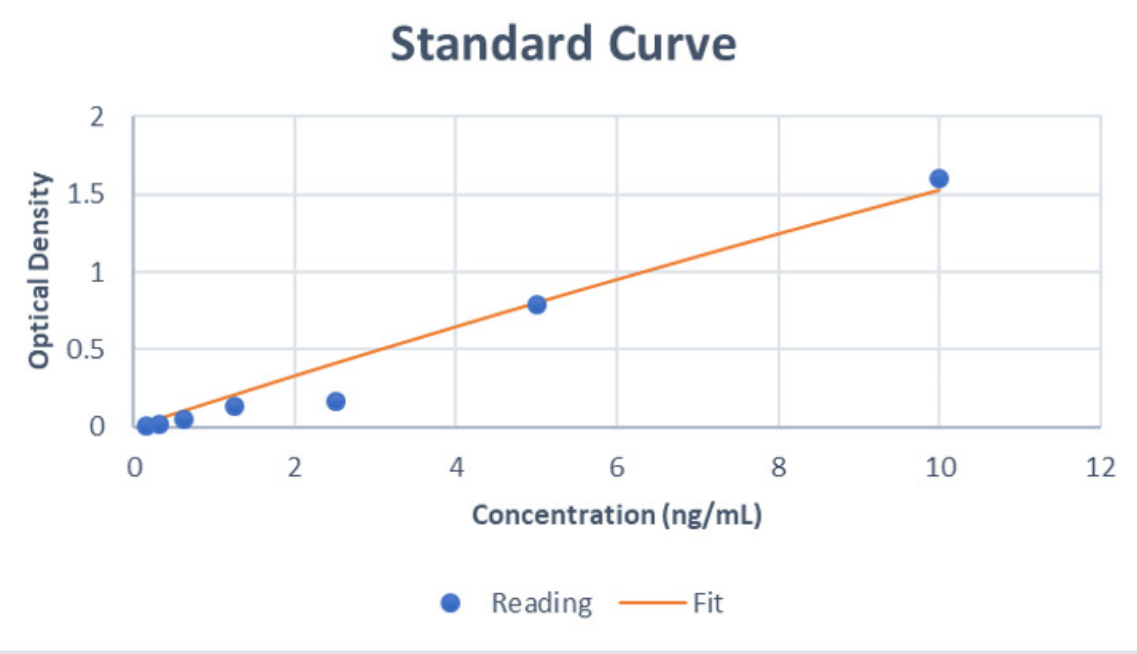

Figure 2: Standard curve of NGAL ELISA assay. 
Calculation of eGFR: The modification of diet in renal disease (MDRD) equation was used for estimating the glomerular filtration rate. It was calculated as follows:

$$
\text { MDRDeGFR } \frac{m L}{\min }=186.3 \times S C r^{-1.154} \times A g e^{-0.23} \times 0.742(\text { forwomen }) \times\left(\frac{1.73}{B S A}\right)
$$

Body Surface Area $(B S A)\left(m^{2}\right)=\sqrt{\frac{h e i g h t \times \text { weight }}{3600}}$

Calculation of Urine Microalbumin Concentration: 30 samples were chosen from the group of patients having $\mathrm{HbA} 1 \mathrm{c}>6 \%$, and these were tested for microalbuminuria through an external laboratory (Al-Arabi Laboratory \& Radiology).

\section{Statistical Analysis}

Data obtained from the experimental analysis and provided by QBB was entered into the statistical software, IBM SPSS for Windows, version 24 and Microsoft Excel 2013. Then, the means and the standard deviations were calculated for the normally distributed continuous data, and one-way analysis of variances (ANOVA) was carried out to detect any significant differences in the mean values of each group. The count with the percentage was calculated for the categorical data. Then, Pearson's correlational analysis was carried out to determine the correlation between uNGAL concentrations and other parameters, keeping NGAL concentration as the dependent factor. If significant correlation was found, then the $\mathrm{R}^{2}$ value was also calculated to determine the degree of correlation, and regression analysis was conducted to determine the dependency of uNGAL concentration on the correlated factors.

\section{Results}

\section{General Study Characteristics}

Since prolonged hyperglycemia precedes DN, HbA1c was considered as a factor to predict probable damage to the kidney. There- fore, the samples were divided into three groups: non-diabetic healthy patients, diabetic patients with $\mathrm{HbA1c}>6$, and diabetic patients with $\mathrm{HbA1c}<6$. As shown in Figure 3. Out of 123 samples, 38 were non-diabetic, 14 were diabetic with $\mathrm{HbA} 1 \mathrm{c}<6 \%$, and 71 were diabetic with $\mathrm{HbA} 1 \mathrm{c}>6 \%$.

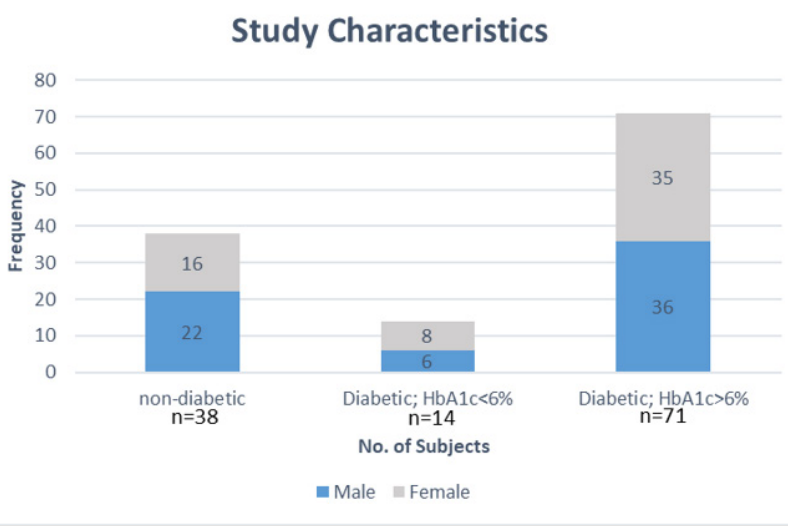

Figure 3: Distribution of patients based on status of diabetes.

\section{Phenotypic Characteristics}

This sample was comprised of 123 subjects having a mean age of 48.24 with an average BMI of $30.57 \mathrm{~kg} / \mathrm{m}^{2}$ and weight of $84.16 \mathrm{~kg}$. An analysis was made using statistical methods (ANOVA) between the three groups. As shown in Table 1, the data demonstrated that there is a statistically significant difference between the mean HbA1c levels of the three groups ( $p=0.00)$. It can also be observed that there is a significant difference in the mean glucose levels $(p=0.00)$ and age of the patients $(p=0.00)$ in each group. However, no statistically significant difference was observed between the mean levels of the other investigated parameters: NGAL, BMI, weight, serum total protein and albumin, eGFR, BUN, SCr and c-peptide of insulin ( $p>0.05$ ).

Table 1: Comparison of mean values of different parameters in each group.

\begin{tabular}{|c|c|c|c|c|}
\hline Variable & Non-Diabetic Patients & $\begin{array}{c}\text { Diabetic Patients with HbA1c } \\
<6 \%\end{array}$ & $\begin{array}{c}\text { Diabetic Patients with HbA1c } \\
\qquad>6 \%\end{array}$ & p-value (2-tailed) \\
\hline HbA1c (\%) & $5.33( \pm 0.31)$ & $5.54( \pm 0.29)$ & $7.85( \pm 1.64)$ & $0.00^{*}$ \\
\hline NGAL (ng/mL) & $14.5( \pm 8.48)$ & $17.2( \pm 8.51)$ & $13.0( \pm 7.51)$ & 0.18 \\
\hline Age (years) & $36.5( \pm 10.8)$ & $42.5( \pm 11.0)$ & $49.5( \pm 9.37)$ & $0.00^{*}$ \\
\hline Weight (kg) & $82.4( \pm 21.1)$ & $77.7( \pm 14.3)$ & $85.2( \pm 17.8)$ & 0.35 \\
\hline $\mathrm{BMI}\left(\mathrm{kg} / \mathrm{m}^{2}\right)$ & $26.8( \pm 9.68)$ & $26.2( \pm 8.38)$ & $29.2( \pm 8.65)$ & 0.29 \\
\hline Glucose (mmol/L) & $5.19( \pm 0.61)$ & $5.56( \pm 1.00)$ & $9.88( \pm 4.39)$ & $0.00^{*}$ \\
\hline Serum Total Protein (g/L) & $73.2( \pm 4.20)$ & $71.0( \pm 3.26)$ & $72.4( \pm 3.98)$ & 0.18 \\
\hline Serum Albumin (g/L) & $45.4( \pm 2.62)$ & $44.0( \pm 1.75)$ & $44.6( \pm 2.81)$ & 0.14 \\
\hline $\mathrm{SCr}(\mathrm{u} / \mathrm{L})$ & $69.1( \pm 14.7)$ & $61.8( \pm 14.5)$ & $62.7( \pm 13.4)$ & 0.06 \\
\hline $\mathrm{BUN}(\mathrm{mmol} / \mathrm{L})$ & $4.18( \pm 1.13)$ & $3.87( \pm 0.85)$ & $4.37( \pm 1.1)$ & 0.26 \\
\hline eGFR (mL/min) & $103.8( \pm 23.9)$ & $109.7( \pm 27.7)$ & $100.9( \pm 22.9)$ & 0.4 \\
\hline c-peptide of Insulin (ng/mL) & $2.47( \pm 1.55)$ & $2.52( \pm 2.31)$ & $2.93( \pm 1.89)$ & 0.4 \\
\hline
\end{tabular}

Note: * - Represents statistical significance $(\mathrm{p}<0.05)$

The data shown here represents the mean and standard deviation. 


\section{Microalbuminuria}

Of the 30 samples selected from the group of diabetic patients having HbA1c> $6 \mathrm{~g} \%$ and tested for albuminuria, 5 patients
(17\%) tested positive for micro/macroalbuminuria, while the rest (83\%) were normoalbuminuric, as demonstrated in Figure 4. Microalbuminuria was characterized as the presence of more than $30 \mathrm{mg}$ of albumin/g of creatinine in urine.

\section{Distribution of Patients* Based on Albuminuria}

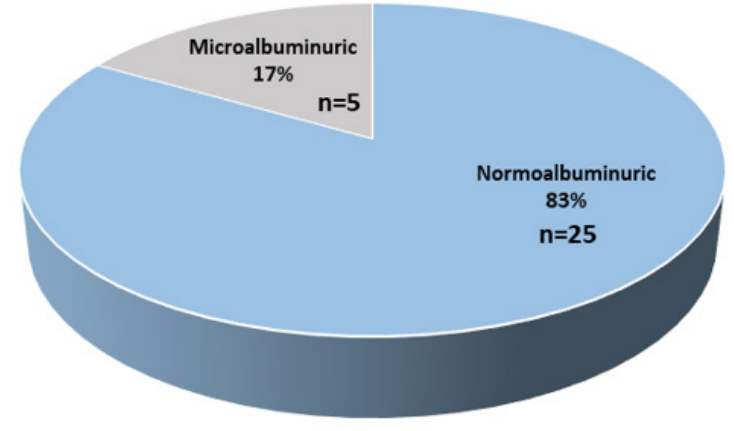

" Normoalbuminuric $=$ Microalbuminuric

Figure 4: Distribution of patients based on albuminuria.

Note: * 30 patients were chosen from the group of diabetic patients with $\mathrm{HbA} 1 \mathrm{c}>6 \%$

\section{Correlational Analysis}

To study the correlation between UNGAL and kidney function, Pearson's correlation factor was calculated, with UNGAL concentration being the dependent factor, and the results are tabulated in Table 2. In the non-diabetic group, no significant correlations were found between UNGAL concentrations and any of the investigated parameters ( $p>0.05$ ), except serum albumin $(\mathrm{p}<0.05)$. Similar to the non-diabetic group, in the Diabetic patients with $\mathrm{HbA} 1 \mathrm{c}<6 \%$ group there was no significant correlations were found between UNGAL concentrations and any of the investigated parameters ( $p>0.05$ ) (Figures 5-7). In Diabetic patients with HbA1c $>6 \%$ group, there was a significant correlation between UNGAL concentrations and glucose $(\mathrm{p}=0.023), \mathrm{HbA1c}(\mathrm{p}=0.026)$ and serum albumin ( $p=0.001)$. Since significant correlation was discovered, the $\mathrm{R}^{2}$ value was also calculated to determine the degree of correlation, and the results are demonstrated in Figures 8 \& 9. It was observed that the degree of correlation was weak $\left(\mathrm{R}^{2}\right.$ values closer to 0$)$.

Table 2: Correlational analysis between uNGAL concentration and other parameters.

\begin{tabular}{|c|c|c|c|}
\hline Variable & Non-Diabetic Patients & Diabetic Patients with HbA1c $<6 \%$ & Diabetic Patients with $\mathrm{HbA1c}>6 \%$ \\
\hline Age (years) & 0.636 & 0.455 & 0.863 \\
\hline Weight (kg) & 0.692 & 0.611 & 0.709 \\
\hline BMI $\left(\mathrm{kg} / \mathrm{m}^{2}\right)$ & 0.415 & 0.56 & 0.329 \\
\hline Glucose (mmol/L) & 0.201 & 0.373 & $0.023^{*}$ \\
\hline HbA1c (\%) & 0.563 & 0.491 & $0.026^{*}$ \\
\hline Serum Total Protein (g/L) & 0.876 & 0.529 & 0.509 \\
\hline Serum Albumin (g/L) & 0.038* & 0.094 & $0.001^{*}$ \\
\hline $\mathrm{SCr}(\mathrm{u} / \mathrm{L})$ & 0.082 & 0.164 & 0.209 \\
\hline BUN (mmol/L) & 0.75 & 0.663 & 0.72 \\
\hline $\mathrm{eGFR}(\mathrm{mL} / \mathrm{min})$ & 0.248 & 0.681 & 0.982 \\
\hline c-peptide of Insulin (ng/mL) & 0.119 & 0.904 & 0.979 \\
\hline c-peptide of Insulin (ng/mL) & $2.47( \pm 1.55)$ & $2.52( \pm 2.31)$ & $2.93( \pm 1.89)$ \\
\hline
\end{tabular}

Note: * - Represents statistical significance $(\mathrm{p}<0.05)$ 


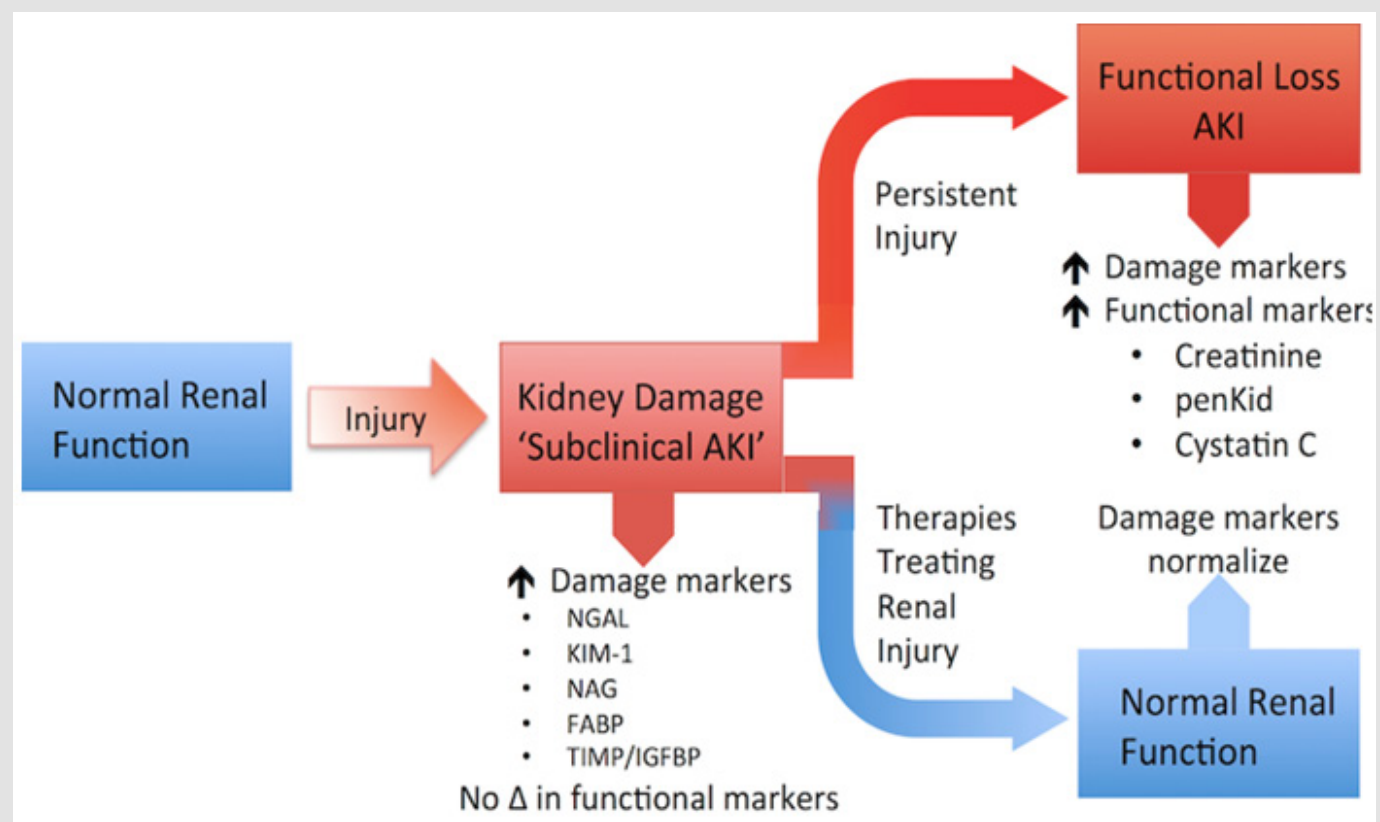

Figure 5: Biomarkers assessing renal function at different stages.

Note: Proposed paradigm of kidney injury with subclinical damage detected by Novel Biomarkers prior to progressing to functional hange; Neutrophil Gelatinase Associated Lipocalin (NGAL) is secreted soon after renal injury. If the injury persists, it leads to the secretion of other damage markers causing functional loss. However, if it gets treated, normal renal function will be restored.

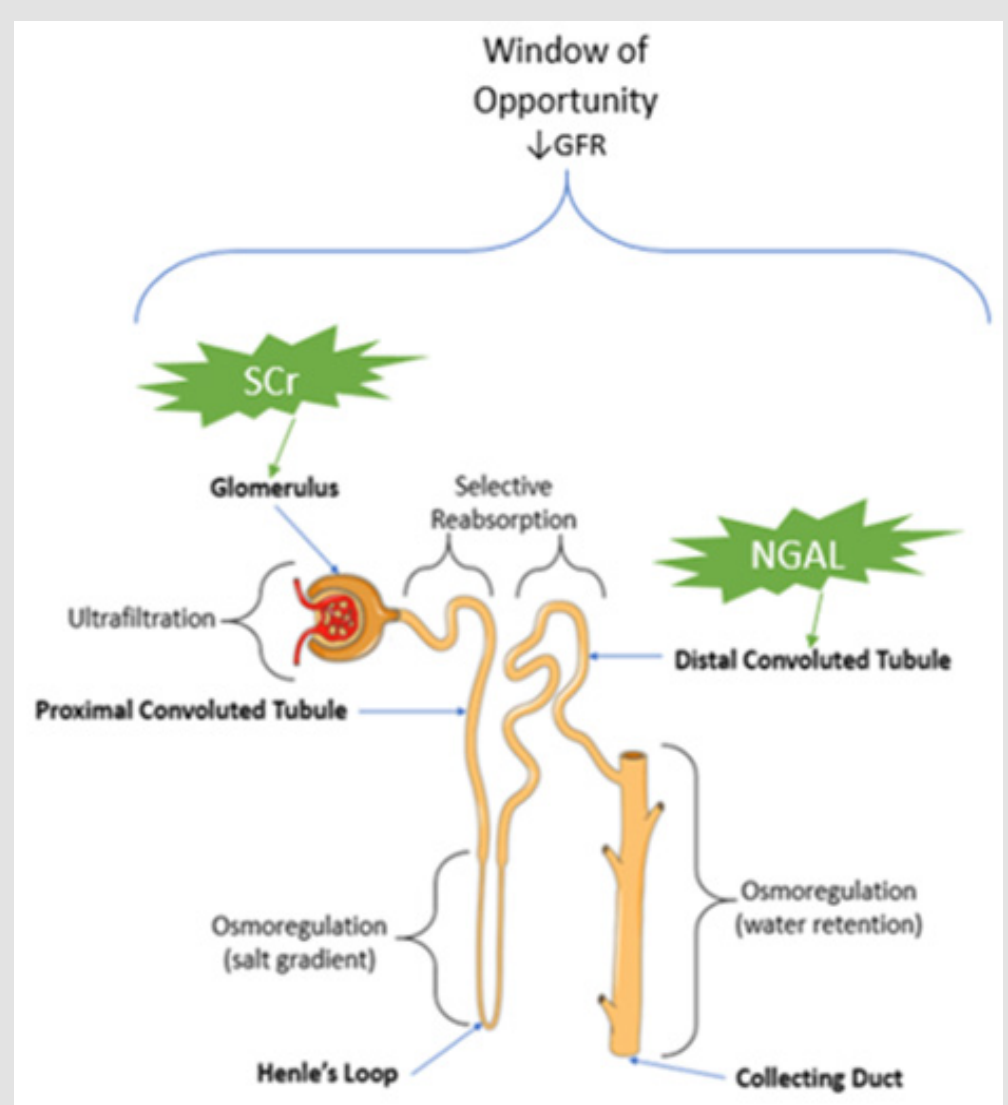

Figure 6: The secretion of NGAL and window of change from kidney injury to kidney failure.

Note: The different anatomical structures of the nephron; Neutrophil Gelatinase Associated Lipocalin (NGAL) is secreted by the distal convoluted tubule in the early stages of diabetic nephropathy (DN) and Serum Creatinine (SCr) is secreted by the glomerulus later. Both are secreted because of kidney damage. 


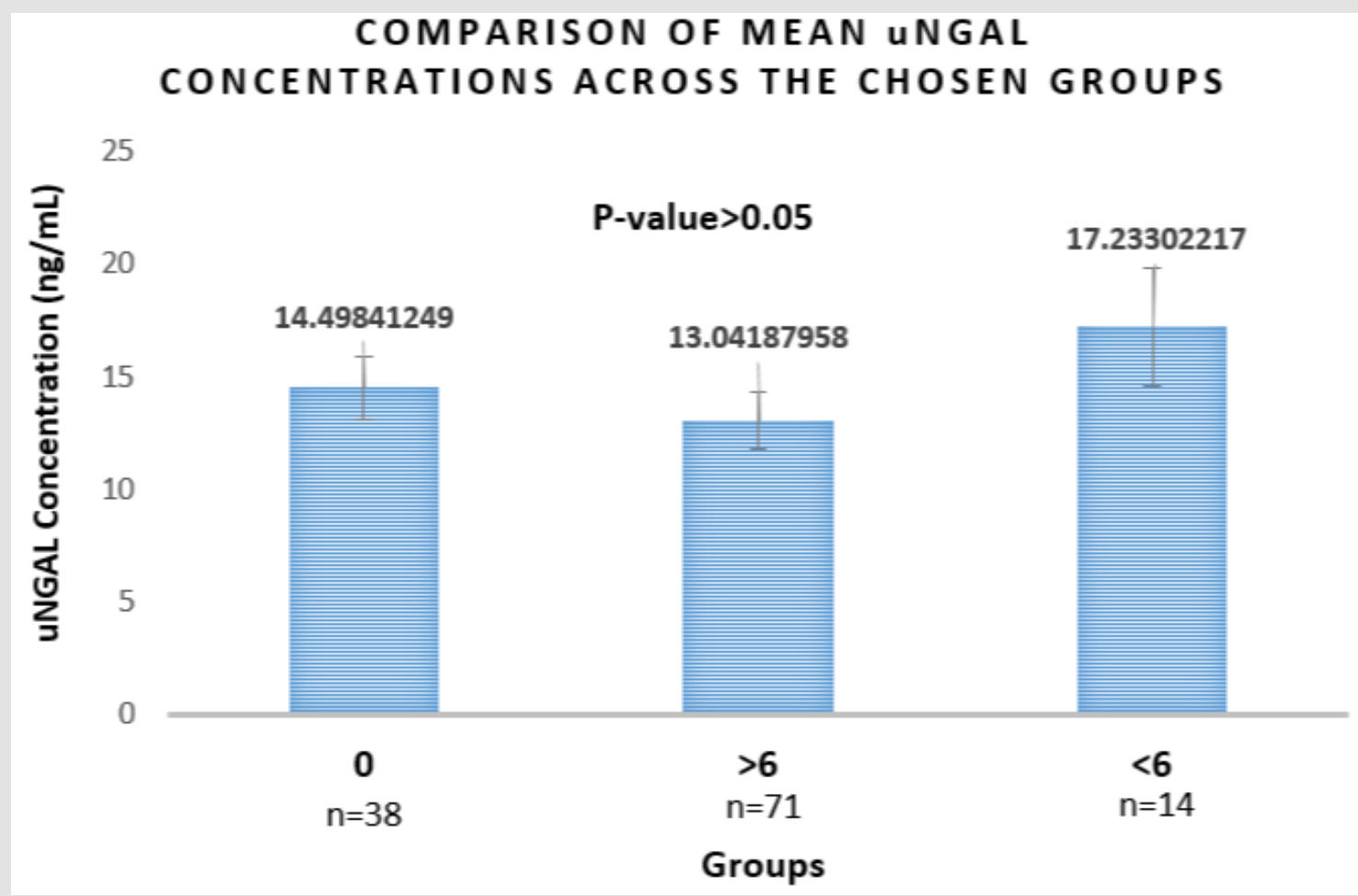

Figure 7: Comparison of mean uNGAL concentrations across the chosen groups.

0 - Healthy non-diabetic subjects

$>6$ - Diabetic patients with $\mathrm{HbA} 1 \mathrm{c}>6 \%$

$<6$ - Diabetic patients with $\mathrm{HbA} 1 \mathrm{c}<6 \%$

\section{Serum Glucose Concentration vs. Urine NGAL Concentration}

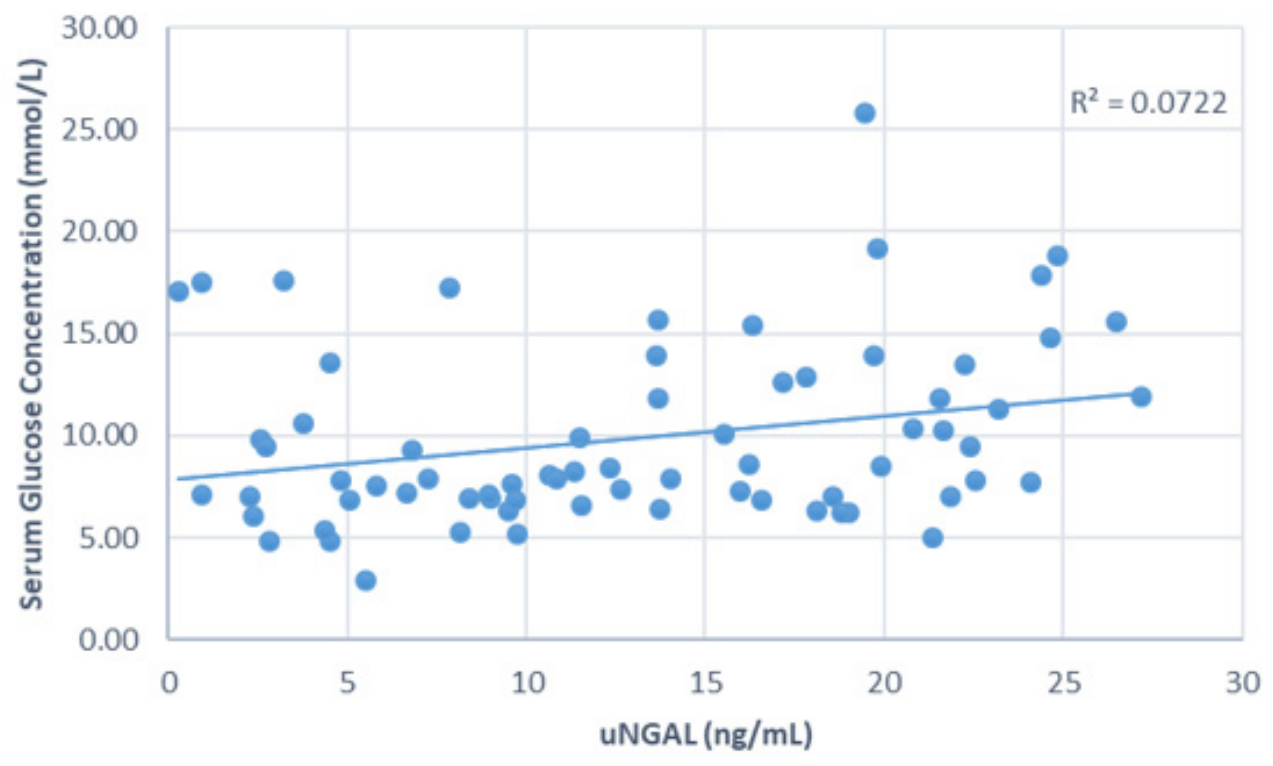

Figure 8: Scatterplot and correlational analysis between serum glucose concentrations and uNGAL concentrations. 


\section{HbA1c \% vs. Urine NGAL Concentration}

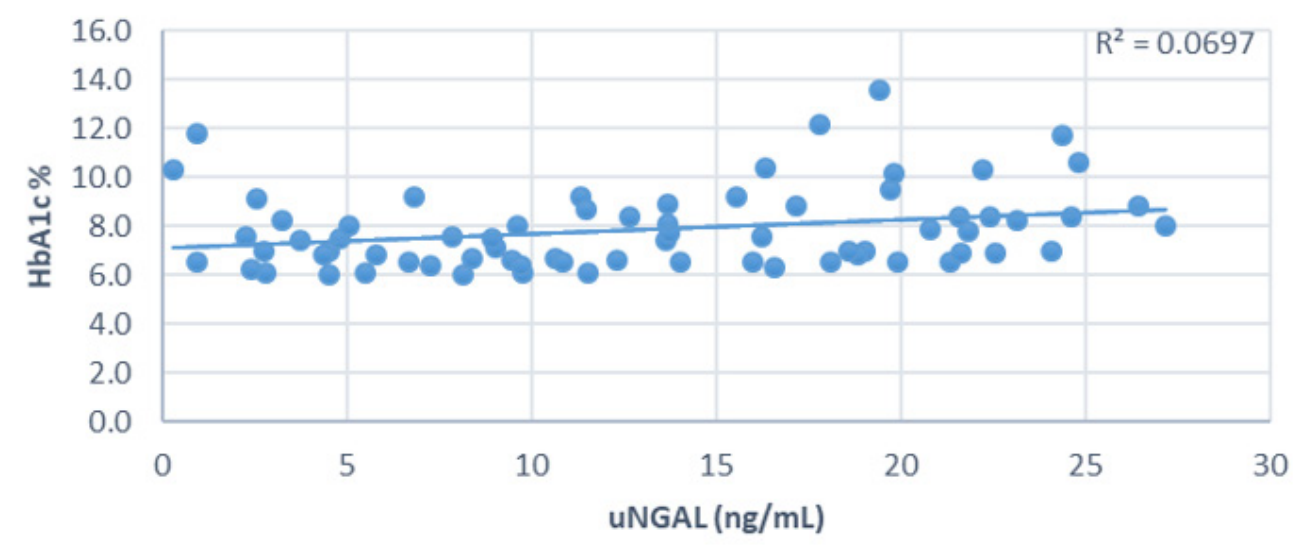

Figure 9: Scatterplot and correlational analysis between $\mathrm{HbA1c} \%$ and uNGAL concentrations.

\section{Discussion}

Diabetes is a growing global epidemic that is expected to affect more than 366 million people (4.4\%) by the year 2030. DN is one of the major complications of diabetes, and it is the largest cause of chronic kidney diseases and end stage renal diseases. To prevent renal failure, it is important to identify a biomarker that can be used to predict the occurrence of DN in its earliest stages. NGAL is considered to be one such promising biomarker. Belonging to the lipocalin family, the monomeric form of this protein is secreted by injured tubular cells of the kidney. In this study, the relationship between urine NGAL concentrations and kidney function was assessed. The uNGAL concentration was measured in 123 subjects, divided into 3 categories: healthy non-diabetic patients (group 1), diabetic patients with $\mathrm{HbA} 1 \mathrm{c}<6 \%$ (group 2), and diabetic patients with $\mathrm{HbA1c}>6 \%$ (group 3 ), as tabulated in Table 1. It was observed that the mean uNGAL concentration was $14.5( \pm 8.48) \mathrm{ng} /$ $\mathrm{mL}, 17.2( \pm 8.51) \mathrm{ng} / \mathrm{mL}, 13.0( \pm 7.51) \mathrm{ng} / \mathrm{mL}$ in groups 1,2 and 3 respectively, although the difference between the groups was not statistically significant ( $p>0.05)$. It is difficult to compare these results to existing literatures discussing uNGAL concentrations in type 2 diabetes, since all the studies have been conducted by comparing nephropathic and non-nephropathic groups [32].

For instance, a study was conducted by Al-Refai and colleagues in 2014 about Egyptian acute-kidney injury subjects, and they discovered a significant difference between healthy subjects and albuminuric patients, but no significant difference was found between normoalbuminuric, microalbuminuric and macroalbuminuric groups [18]. Moreover, a study conducted by Lacquaniti and colleagues in 2013 on Italian Type 1 diabetic patients demonstrated a significant difference in the mean levels of uNGAL between healthy patients and normoalbuminuric type 1 patients [33]. The difference in results may be due to the difference in sample size, the type of diabetes, the type of kidney injury or the difference in ethnicities. To investigate the relationship between uNGAL concentrations and the kidney function, correlational analysis (Pearson's correlation) was conducted between uNGAL concentrations and the kidney function tests: SCr, BUN and eGFR, along with the HbA1c, and fasting glucose concentration. It was observed that in groups 1 (healthy) and 2 (diabetic, HbA1c<6\%), uNGAL concentrations showed no significant correlation with any parameter. However, in group 3 (diabetic, HbA1c $>6 \%$ ), significant correlation was observed between uNGAL concentrations with $\mathrm{HbA} 1 \mathrm{c}$ and glucose concentration $(\mathrm{p}<0.05)$, and it was a weak positive correlation $\left(\mathrm{R}^{2}=0.06\right.$ and 0.07 respectively). This implies that uNGAL concentrations are secreted in larger amounts following prolonged hyperglycemia, which may be related to the pathogenesis of $\mathrm{DN}$.

Moreover, significant weak negative correlation was observed between UNGAL and serum albumin concentrations $(\mathrm{p}<0.05$ and $\mathrm{R}^{2}=0.0007$ ), which means that as serum albumin decreases, uNGAL concentration increases. Decrease in serum albumin is often due to its increased loss in urine. Therefore, it can be inferred that there might be positive correlation between UNGAL and urine albumin concentrations, which has been reported by Papadopoulou-Marketou and colleagues (2017), among others [34]. The discrepant results could be attributed to the small sample size in this study and the variation in age, ethnicity and stage of the disease, and no conclusion can be drawn from these results. Moreover, it should be taken into consideration that the complications of diabetes and prolonged hyperglycemia can be other microvascular complications, such as retinopathy and neuropathy, or macrovascular complications, such as atherosclerosis, cardiovascular disease and coronary heart disease, among others. Since enough clinical information about the patients were not available, it was difficult to confirm the 
status of the other patients in group 3 (diabetic; HbA1c $>6 \%$ ). Since this study tries to correlate disease development with structural involvement through secretion of a protein, it would be more beneficial if the study design was of the observational or prospective format, instead of the cross-sectional format used. Moreover, it would prove useful to test the hypothesis on a larger sample size.

\section{Acknowledgement}

We would like to extend our gratitude to Ms Shazda Cowdhury and Ms. Horia El-Sherif, Biomedical Sciences Students, College of Health Sciences at Qatar University for helping us in the practical and statistical analysis and interpretation. Thanks to Ms. Mooza AlKhinji, Senior Lab Technician, College of Health Sciences at Qatar University for assisting us with experimental work.

\section{Compliance with Ethical Standards}

The experimental design including participation of human subjects were obtained from the Qatar Biobank (QBB), under the project no. QBB_IRB_Ex-2017-RES-ACC-0091-0038.

\section{Author Contributions}

Elham Sharif (corresponding author) designed and supervised the project. Mariam Alwakeel equally contributed to data collection, data analysis, and manuscript draft. All authors equally contributed to the final editing and revision of the manuscript.

\section{References}

1. (2017) International Diabetes Federation. IDF Diabetes Atlas, $\left(8^{\text {th }}\right.$ Edn). Brussels, Belgium.

2. Haj Bakri A, Al Thani A (2013) Chronic Disease Risk Factor Surveillance: Qatar STEPS Report 2012 (Rep.). Ministry of Health.

3. Oltean S, Coward R, Collino M, Baelde H (2017) Diabetic Nephropathy: Novel Molecular Mechanisms and Therapeutic Avenues. BioMed Research International p. 1.

4. Perkins BA, Ficociello LH, Roshan B, Warram JH, Krolewski AS (2010) In patients with type 1 diabetes and new-onset microalbuminuria the development of advanced chronic kidney disease may not require progression to proteinuria. Kidney International 77(1): 57-64.

5. Thomas M, Burns W, Cooper M (2005) Tubular changes in early diabetic nephropathy. Advances in Chronic Kidney Disease 12(2): 177-186.

6. Yang Y, He X, Chen S, Wang L, Li E, et al. (2009) Changes of serum and urine neutrophil gelatinase-associated lipocalin in type-2 diabetic patients with nephropathy: one year observational follow-up study. Endocrine 36(1): 45-51.

7. Firu SG, Streba CT, Firu D, Tache DE, Rogoveanu I (2015) Neutrophil Gelatinase Associated Lipocalin (NGAL) - a biomarker of renal dysfunction in patients with liver cirrhosis: Do we have enough proof? Journal of medicine and life p. 15-20.

8. (2016) World Health Organization. Global report on diabetes (Rep.).

9. (2012) Health, S.C.o. Qatar Stepwise Report. Chronic Disease Risk Factor Surveillance 2013. Supreme Council of Health, Qatar pp. 124.

10. Awad Susanne F, O’Flaherty M, Critchley J, Abu Raddad LJ (2017) Forecasting the burden of type 2 diabetes mellitus in Qatar to 2050: A novel modeling approach. Diabetes Research and Clinical Practice 137: 100-108.
11. Olokoba AB, Obateru OA, Olokoba LB (2012) Type 2 diabetes mellitus: a review of current trends. Oman medical journal 27(4): 269-273.

12. Magri CJ, Fava S (2009) The role of tubular injury in diabetic nephropathy. European Journal of Internal Medicine 20(6): 551-555.

13. Thomas M, Burns W, Cooper M (2005) Tubular changes in early diabetic nephropathy. Advances in Chronic Kidney Disease 12(2): 177-186.

14. Devarajan P (2008) Neutrophil gelatinase-associated lipocalin (NGAL): A new marker of kidney disease. Scandinavian Journal of Clinical and Laboratory Investigation 241: 89-94.

15. Dabla PK (2010) Renal function in diabetic nephropathy. World Journal of Diabetes 1(2): 48-56.

16. Cruz DN, Cal MD, Garzotto F, Perazella MA, Lentini P, et al. (2009) Plasma neutrophil gelatinase-associated lipocalin is an early biomarker for acute kidney injury in an adult ICU population. Intensive Care 36(3): 444-451.

17. Fiseha $T$ (2015) Urinary biomarkers for early diabetic nephropathy in type 2 diabetic patients. Biomarker Research 3: 16.

18. Al Refai AA, Tayel SI, Ragheb A, Dala AG, Zahran A (2014) Urinary Neutrophil Gelatinase Associated Lipocalin as a Marker of Tubular Damage in Type 2 Diabetic Patients with and without Albuminuria. Open Journal of Nephrology 4(1): 37-46.

19. Mori K, Nakao K (2007) Neutrophil gelatinase-associated lipocalin as the real-time indicator of active kidney damage. Kidney International 71(10): 967-970.

20. Devarajan P (2010) Review: Neutrophil gelatinase-associated lipocalin: A troponin-like biomarker for human acute kidney injury. Nephrology 15(4): 419-428.

21. Perkins BA, Aiello LP, Krolewski AS (2009) Diabetes complications and the renin-angiotensin system. New England Journal of Medicine 361(1): 83-85.

22. Gluck C, Ko Y, Susztak K (2017) Precision Medicine Approaches to Diabetic Kidney Disease: Tissue as an Issue. Current Diabetes Reports 17(5): 30 .

23. Hosten AO (1990) BUN and creatinine. In Clinical Methods: The History, Physical, and Laboratory Examinations ( $3^{\text {rd }}$ Edn.) Butterworths, Boston, USA.

24. Ding H, He Y, Li K, Yang J, Li X, et al. (2007) Urinary neutrophil gelatinase-associated lipocalin (NGAL) is an early biomarker for renal tubulointerstitial injury in IgA nephropathy. Clinical Immunology 123(2): 227-234.

25. Schmidtott KM, Mori K, Li JY, Kalandadze A, Cohen DJ, et al. (2007) Dual action of neutrophil gelatinase-associated lipocalin. J Am Soc Nephrol 18(2): 407-413.

26. Ahn H, Hyun C (2013) Evaluation of serum neutrophil gelatinaseassociated lipocalin (NGAL) activity in dogs with chronic kidney disease. Veterinary Record 173(18): 452.

27. Magnusson NE, Hornum M, Jørgensen KA, Hansen JM, Bistrup C, et al. (2012) Plasma neutrophil gelatinase associated lipocalin (NGAL) is associated with kidney function in uraemic patients before and after kidney transplantation. BMC Nephrology 13(1).

28. Assal HS, Tawfeeq S, Rasheed EA, El Lebedy D, et al. (2013) Serum Cystatin C and Tubular Urinary Enzymes as Biomarkers of Renal Dysfunction in Type 2 Diabetes Mellitus. Clin Med Insights Endocrinol Diabetes 6: 7-13.

29. Nielsen SE, Schjoedt KJ, Astrup AS, Tarnow L, Lajer M, et al. (2010) Neutrophil Gelatinase-Associated Lipocalin (NGAL) and Kidney Injury Molecule 1 (KIM1) in patients with diabetic nephropathy: a crosssectional study and the effects of lisinopril. Diabetic Medicine 27(10): 1144-1150. 
30. Liu F, Yang H, Chen H, Zhang M, Ma Q (2015) High expression of neutrophil gelatinase-associated lipocalin (NGAL) in the kidney proximal tubules of diabetic rats. Advances in Medical Sciences 60(1): 133-138.

31. Wu J, Ding Y, Zhu C, Shao X, Zou D (2013) Changes of urine neutrophil gelatinase associated lipocalin in patients with diabetic nephropathy and the related clinical significance. Academic Journal of Second Military Medical University 32(9): 1032-1033.

32. Human Lipocalin-2/NGAL Quantikine ELISA Kit. (n.d.).

ISSN: 2574-1241

DOI: 10.26717/BJSTR.2019.22.003692

Elham Sharif. Biomed J Sci \& Tech Res

(c) (i) This work is licensed under Creative

Submission Link: https://biomedres.us/submit-manuscript.php
33. Lacquaniti A, Donato V, Pintaudi B, Vieste GD, Chirico V, et al. (2013). "Normoalbuminuric" diabetic nephropathy: Tubular damage and NGAL. Acta Diabetologica 50(6): 935-942.

34. Papadopoulou Marketou N, Skevaki C, Kosteria I, Peppa M, Chrousos G, Papassotiriou I, et al. (2014) NGAL and cystatin C: two possible early markers of diabetic nephropathy in young patients with type 1 diabetes mellitus: one year follow up. Hormones 14(2): 232-240.

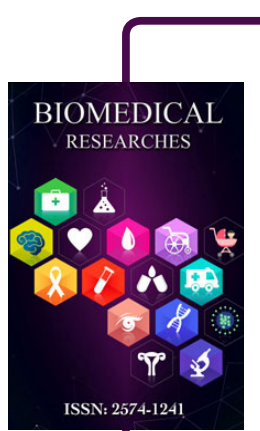

Assets of Publishing with us

- Global archiving of articles

- Immediate, unrestricted online access

- Rigorous Peer Review Process

- Authors Retain Copyrights

- Unique DOI for all articles

https://biomedres.us/ 